\title{
Application of a three-dimensional (3D) breast cancer model to study macrophage polarization
}

\author{
AGATA GOLABEK ${ }^{1}$, MARIUSZ KACZMAREK ${ }^{1,2}$, EWELINA DONDAJEWSKA ${ }^{3}$, \\ KOSMA SAKRAJDA $^{1}$, ANDRZEJ MACKIEWICZ ${ }^{1,3}$ and HANNA DAMS-KOZLOWSKA ${ }^{1,3}$ \\ ${ }^{1}$ Department of Diagnostics and Cancer Immunology, Greater Poland Cancer Centre, 61-866 Poznan; \\ ${ }^{2}$ Department of Immunology, Poznan University of Medical Sciences, 60-806 Poznan, \\ ${ }^{3}$ Department of Cancer Immunology, Poznan University of Medical Sciences, 61-701 Poznan, Poland
}

Received December 24, 2019; Accepted February 4, 2021

DOI: $10.3892 /$ etm.2021.9913

\begin{abstract}
Knowledge of the tumor microenvironment is crucial for developing an effective strategy to treat cancer. Recently, anticancer therapies targeting macrophages have been intensively investigated. Increased understanding of the importance of the tumor microenvironment has led to the development of three-dimensional (3D) in vitro tumor models. However, established techniques for studying tumor-associated macrophages in vitro are limited. We have previously characterized a 3D breast cancer model consisting of breast cancer cells and fibroblasts cocultured on a silk scaffold. In the present study, the influence of this model on macrophage polarization was investigated. The expression of macrophage markers was studied using reverse transcription-quantitative PCR and flow cytometry. The activity of nitric oxide synthase and arginase in macrophages was also measured. The presented model appeared to induce the polarization of macrophages towards an M2 phenotype. In this 3D tumor model, the in vivo behavior of macrophages could be reproduced. This model may be beneficial for the study of tumor biology and for screening drugs.
\end{abstract}

\section{Introduction}

The tumor microenvironment contributes to cancer growth, invasion and progression $(1,2)$. At all stages of neoplastic development, cancer cells interact with surrounding cellular infiltrates, and vice versa, resulting in the generation of cells with a wide spectrum of activation states. Cancer-associated fibroblasts (CAFs), myofibroblasts, inflammatory immune

Correspondence to: Dr Hanna Dams-Kozlowska, Department of Diagnostics and Cancer Immunology, Greater Poland Cancer Centre, 15 Garbary Street, 61-866 Poznan, Poland

E-mail: hanna.dams-kozlowska@wco.pl

Key words: cancer, three-dimensional model, silk scaffold, tumor-associated macrophages, tumor microenvironment, cancer immunotherapy cells, adipocytes, epithelial cells, endothelial cells and pericytes communicate with cancer cells via cell-cell contact-dependent mechanisms and soluble factors (3-5). Among the immune cells recruited to the tumor development site, macrophages are a particularly relevant population (6).

Macrophages are an indispensable population of immune cells that maintain the homeostasis of the body and are engaged in the course of diseases. They are found in all tissues and are characterized by their complexity and multilateral function (7). Depending on the signals received, macrophages can develop distinct phenotypic programs through a process known as polarization towards either the M1 (classical) or the M2 (alternative) phenotype (7). By contrast to classical macrophage activation, which is associated with the development of an inflammatory reaction, alternative activation is related to inhibition of the effector phase during the immune response (8). Similarly, at the tumor site, tumor-associated macrophages (TAMs) can undergo polarization $(9,10)$. At the early stages of cancer, TAMs assume an M1-like phenotype, but during disease progression, the local signals derived from cancer cells, stromal cells, inflammatory cells and hypoxia promote the polarization of TAMs towards an M2-like phenotype (9). Classically activated M1 macrophages in the tumor environment serve an antiangiogenic and antitumorigenic role (11). At the advanced stage of cancer, M2-like macrophages, which constitute the majority of the TAM population, promote tumor growth by contributing to immunosuppression, angiogenesis and chronic inflammation $(11,12)$.

Current understanding of macrophage polarization is largely based on in vitro studies. Classical activation of macrophages is stimulated by proinflammatory signaling molecules, such as the cytokines interferon (IFN) $-\gamma$ or tumor necrosis factor (TNF), and can also develop in response to exposure to microbes or microbial products such as lipopolysaccharide (LPS) (7). Such stimulation activates the inflammatory mechanism that leads to increased secretion of proinflammatory cytokines [e.g. TNF, IFN- $\beta$, interleukin (IL)-12, IL-6 and IL-1 $\beta$ ] and chemokines [e.g., C-C motif chemokine ligand 2 (CCL2), also known as monocyte chemoattractant protein-1 (MCP-1) and C-X-C motif chemokine (CXCL)-10 and CXCL11] and to upregulation of surface molecules, such as major histocompatibility complex (MHC) class II 
(I-A/I-E) and CD86 costimulatory molecules (13). Activated M1 macrophages have an enhanced ability to secrete reactive nitrogen species (RNS) and oxygen species, which exert toxic effects on cancer cells (13).

Activation of the macrophage M2 phenotype is stimulated by cytokines IL-4, IL-10, IL-13 and macrophage-colony stimulation factor $(14,15)$. The M2 phenotype is associated with an expression profile of IL-12 $2^{\text {low }}$, IL-2 $3^{\text {low }}$ and IL-10 $0^{\text {high }}$ and with high production of profibrotic factors such as fibronectin, matrix metalloproteinases (MMPs), IL-1 $\beta$ and transforming growth factor (TGF)- $\beta$ (16). The most recognized markers of human M2 macrophages are scavenger receptor- 1 class A (CD204, also known as SR-AI or MSR1), mannose receptor-1 (CD206), the hemoglobin scavenger receptor (CD163) and macrophage galactose-type C-type lectin (MGL; CD301a) (17-19). In mice, M2 profile markers include arginase 1 (Arg1), resistin-like molecule $\alpha$ (Fizz-1) and chitinase-like protein (19). Additionally, M2 TAMs secrete vascular endothelial growth factor (VEGF), MMPs, epidermal growth factor, TGF- $\beta$, IL-10 and CCL2 (20). Immunosuppressive mediators such as IL-10, TGF- $\beta$ and reactive nitrogen species, which are released by M2 TAMs, suppress T cells proliferation and reduce the antigen-presenting capacity and antitumor response of immunological cells, contributing directly to immune evasion by cancer cells (21).

As macrophages (both M1 and M2) serve a substantial role in tumor development, it is crucial to understand the interaction between macrophages and other cell types in the tumor microenvironment. However, most of the in vitro models generated to investigate the interactions between cancer cells and macrophages are based on two-dimensional (2D) coculture of these cells or the application of conditioned media obtained from cancer cells in 2D culture $(22,23)$. Several studies have examined the regulatory mechanisms underlying macrophage polarization in the tumor microenvironment using a Transwell system, where a porous membrane separates flat cultures of two cell types and cells can thus interact via paracrine signaling $(24,25)$. However, flat $2 \mathrm{D}$ cell culture does not mimic the conditions that occur in the body (26). To address this issue, various models of three-dimensional (3D) culture, including microfluidics-based devices, scaffolds and hanging drop technologies, have been proposed $(27,28)$. These models, which enable spatial cell growth, more accurately reproduce the three-dimensional natural microenvironment (27). Due to these approaches, many interactions between cancer cells and cells surrounding the tumor have been demonstrated (29). Although a number of reports are available (30-32), there is a limited amount of data describing $3 \mathrm{D}$ in vitro techniques for studying macrophage polarization in the cancer microenvironment. To address this issue, the present study investigated the influence of a 3D cancer model established in our lab on the status of macrophages.

The 3D in vitro model of breast cancer consists of a spatial coculture of cancer cells and fibroblasts on a silkworm silk scaffold (33). The porosity of the silk scaffold has been optimized to facilitate the growth of cancer cells. Based on the characteristics of the kinetics of cell growth, salt-leached scaffolds with a pore diameter of 250-500 $\mu \mathrm{m}$ were selected to generate a 3D cancer model. To describe this model, the drug cytotoxicity, growth kinetics, morphology and gene expression profile of cocultured cells was investigated and these profiles were compared with cells cultured in a $2 \mathrm{D}$ system and in monoculture on the 3D scaffold. Culture in the 3D coculture system caused a modification of the cells' morphology and significantly increased the production of extracellular matrix (ECM) (33). The interaction of cocultured cells and their spatial growth also induced cellular changes related to epithelial-mesenchymal transition (EMT) and cancer-associated fibroblast markers (33). Moreover, dynamic culture conditions were recently introduced to the $3 \mathrm{D}$ model. The implementation of culture medium flow enabled the induction of shear forces into the system which more closely reflects the conditions observed in vivo (34).

In the present study, a functional assessment of the obtained 3D model of breast cancer was performed through analysis of the effect of its microenvironment on macrophages. The conditioned media collected from the 3D cancer model and control 3D cell cultures were used to stimulate macrophages and the phenotypic status of the macrophages was then examined. By using a silk-based 3D breast cancer model the in vivo behavior of macrophages could be reproduced.

\section{Materials and methods}

Establishment of a $3 D$ cancer model and preparation of conditioned media (CM). The EMT6 mouse breast cancer cells and NIH3T3 mouse fibroblasts were obtained from American Type Culture Collection and modified to express green fluorescence protein (GFP) and far-red fluorescence protein (turboFP635), respectively (EMT6/GFP and NIH3T3/635, respectively). The indicated cell modifications were described previously (33). Cells were maintained in DMEM (Sigma-Aldrich; Merk KGaA) supplemented with 10\% FBS (Sigma-Aldrich; Merck KGaA) and $80 \mu \mathrm{g} / \mathrm{ml}$ gentamycin (KrKa, d.d. Novo Mesto). Cells were grown at $37^{\circ} \mathrm{C}$ in a humidified atmosphere containing $5 \% \mathrm{CO}_{2}$.

Bombyx mori silkworm cocoons were obtained from the Institute of Natural Fibers and Medicinal Plants (Poznan, Poland) and the silk fibroin solution was extracted as described previously (33). Porous scaffolds were prepared with a salt leaching technique by pouring $0.5 \mathrm{ml}$ silk fibroin solution $(\sim 8 \% \mathrm{w} / \mathrm{v})$ into a polyethylene container $2 \mathrm{~cm}$ in diameter and adding $1 \mathrm{~g}$ sodium chloride crystals (Thermo Fisher Scientific Inc.) of size 250-500 $\mu \mathrm{m}$ (33). Sodium chloride of the defined particle size was obtained by sieving through 250 and $500 \mu \mathrm{m}$ test sieves (33).

A total of $3 \times 10^{5}$ EMT6/GFP and NIH3T3/635 cells were seeded on the scaffold at a ratio of 1:9, respectively, as described previously (33). After 2 days, $3 \times 10^{5}$ EMT6/GFP cells or NIH3T3/635 cells were seeded on separate scaffolds. After 7 or 5 days, scaffolds of cocultured and monocultured cells of both lines were transferred into fresh wells of 24-well plates with 3 (unless otherwise specified) scaffolds of each culture type in each well. Next, the cells were incubated at $37^{\circ} \mathrm{C}$ in fresh medium for $24 \mathrm{~h}$ under standard culture conditions. After $24 \mathrm{~h}, \mathrm{CM}$ was collected and filtered through $0.45 \mu \mathrm{m}$ filters.

Macrophage activation. A total of $1 \times 10^{6} \mathrm{~J} 774$ cells (American Type Culture Collection) were seeded in 6-well plates and cultured at $37^{\circ} \mathrm{C}$ for $24 \mathrm{~h}$ in DMEM medium supplemented 


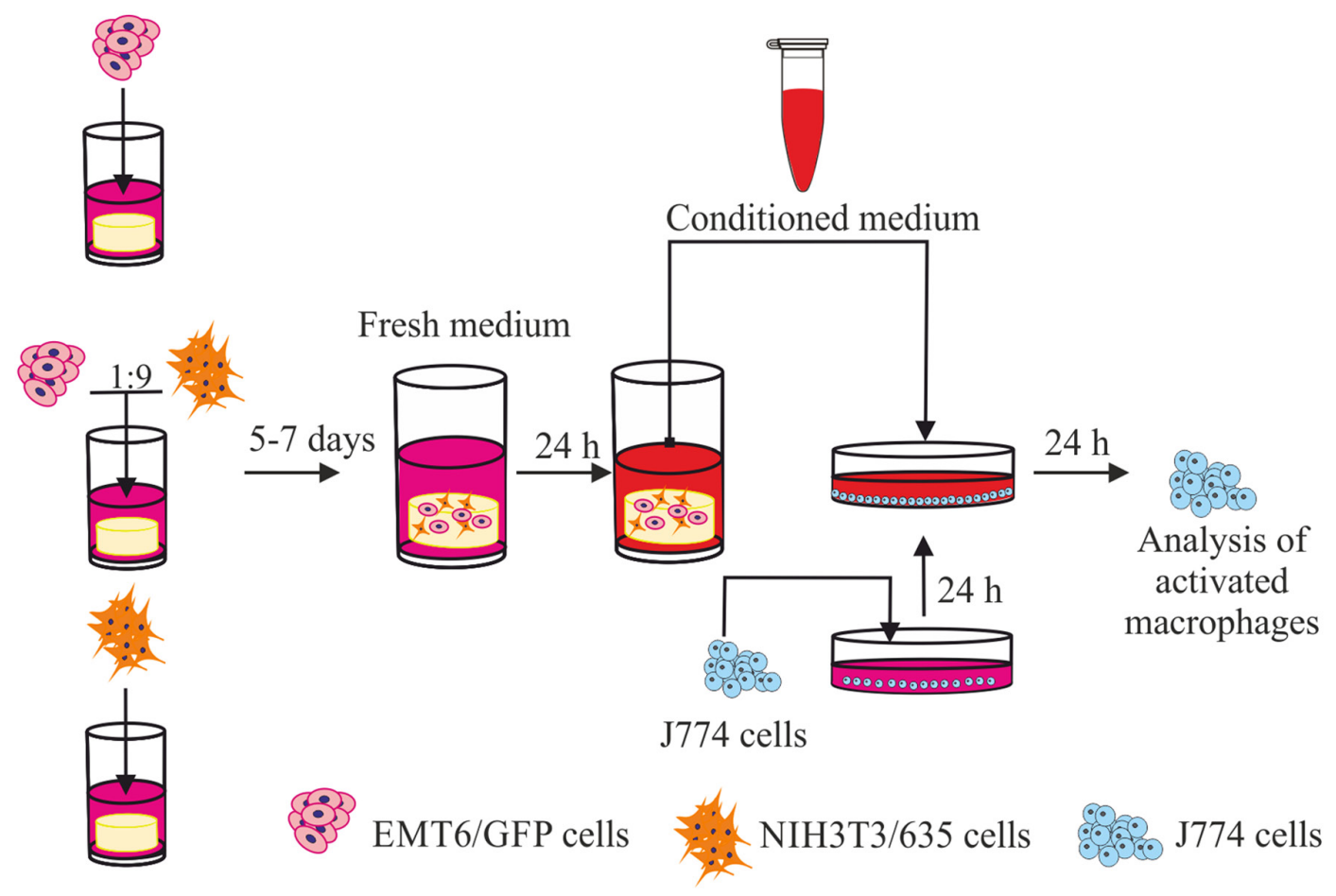

Figure 1. Schematic representation of the experimental protocol. In brief, EMT6/GFP and NIH3T3/635 cells were seeded on scaffolds as follows: i) EMT6/GFP cell monoculture (EMT6); ii) NIH3T3/635 cell monoculture (3T3); and iii) EMT6/GFP and NIH3T3/635 cell coculture (MIX, 3D breast cancer model). Cells were cultured for 5-7 days, until the number of cells in each culture system was approximately 1 million. Subsequently, scaffolds were transferred into a new separate well and incubated at $37^{\circ} \mathrm{C}$ for $24 \mathrm{~h}$ in fresh medium. The following day, conditioned media were collected and added to J774 macrophages and macrophages were analyzed after an additional $24 \mathrm{~h}$. GFP, green fluorescent protein.

with $10 \%$ FBS and $80 \mu \mathrm{g} / \mathrm{ml}$ gentamycin. The following day, the medium was removed and replaced with $1.7 \mathrm{ml}$ of $\mathrm{CM}$ and incubated at $37^{\circ} \mathrm{C}$ for an additional $24 \mathrm{~h}$ (unless otherwise specified). J774 cells cultured in $1.7 \mathrm{ml}$ of DMEM medium was the negative control. The sensibility control (positive control for cell reactivity) was J774 cells cultured in culture medium supplemented with mouse IL- 4 and IL- 6 at a final concentration of $50 \mathrm{ng} / \mathrm{ml}$ each.

Fig. 1 provides a schematic representation of the experimental protocol consisting of the establishment of 3D cell cultures, CM preparation and macrophage activation.

$R N A$ isolation and reverse transcription-quantitative PCR (RT-qPCR) analysis. Macrophages were stimulated for $12 \mathrm{~h}$ with collected CM. Next, RNA was isolated from J774 cells using TRI reagent (Sigma-Aldrich; Merck KGaA) following the manufacturer's protocol. The quality and quantity of RNA were determined spectrophotometrically. High-quality RNA was reverse transcribed using the iScriptcDNA synthesis kit (Bio-Rad Laboratories, Inc.) in accordance with the manufacturer's protocol. Expression of Itgax, Mrcl, Ccl24, RetnIa, Argl, Illo, Vegfa, Il6, Cd44, Tgfbl, Tnfa and Ki67 genes was analyzed via RT-qPCR. Reactions were designed to utilize fluorescent hydrolysis probes (Universal Probe Library; Roche Diagnostics) specific towards the expected products. The used gene-specific primers and fluorescent probes are listed in Table I. The primers were designed at the Universal Probe Library Assay Design Center (https://lifescience.roche.com/en_pl/articles/Universa 1-ProbeLibrary-System-Assay-Design.html) The reaction was carried out on LightCycler 480 (Roche Diagnostics) and using a Probes Master kit (cat. no. 04707494001; Roche Diagnostics) according to manufacturer's protocol. Gene expression was normalized to $T u b b$ ( $\beta$-tubulin) expression for each sample (Table I). Fold gene expression changes were calculated using the $2^{-\Delta \Delta C q}$ method (35). The experiment was repeated at least three times in triplicate.

Quantitation of cytokine/chemokine levels by cytometric bead array. A mouse inflammatory cytometric bead array (CBA) kit (cat. no. 552364; BD Biosciences) was used to determine the concentrations of IL-12p70, TNF $\alpha$, IFN- $\gamma$, MCP-1, IL-10 and IL-6 in CM. Briefly, a mixture of six capture bead populations $(50 \mu \mathrm{l})$ with distinct fluorescence intensities and precoated with capture antibodies specific for the abovementioned proteins was added to $50 \mu 1$ of the sample. Next, PE-conjugated detection antibodies $(50 \mu \mathrm{l})$ were added to each sample and incubated for $2 \mathrm{~h}$ in the dark at room temperature. The unbound antibodies were removed by the addition of $1 \mathrm{ml}$ of wash buffer followed by centrifugation at room temperature ( $200 \mathrm{x}$ g for $5 \mathrm{~min}$ ). Next, the captured beads were resuspended in wash buffer $(300 \mu \mathrm{l})$ and analyzed using a FACSAriaII flow cytometer (BD Biosciences) and FACSDiva v6.1.2 software (BD Biosciences). The amount of each cytokine in the CM was calculated based on the corresponding standard curve with FCAP v3.0 software (BD Biosciences). The experiment was repeated three times.

Analysis of the activated macrophages by flow cytometry. J774 cells were detached from the plate using a cell scraper, washed three times in PBS and analyzed using a FACSAria 
Table I. List of primers and corresponding probes used for a reverse transcription-quantitative PCR.

\begin{tabular}{|c|c|c|}
\hline Gene (protein) name & Primer sequence $\left(5^{\prime}-3^{\prime}\right)$ & UPL Probe no \\
\hline \multirow[t]{2}{*}{ Tubb ( $\beta$-tubulin $)$} & F: GCTGGACCGAATCTCTGTGT & \#95 \\
\hline & R: GACCTGAGCGAACGGAGTC & \\
\hline \multirow[t]{2}{*}{ Itgax (CD11c) } & F: GAGAAGATCTTTGCCATTGAGG & \#79 \\
\hline & R: CAGAACTGGTCCATCAGGTG & \\
\hline \multirow[t]{2}{*}{$\operatorname{Mrc1}(\mathrm{CD} 206)$} & F: GATGGAACCCCAGTGACATT & \#80 \\
\hline & R: TGTCCGCCCAGTATCCAT & \\
\hline \multirow{2}{*}{ Retnla (Fizz-1) } & F: GCACTAGTGTCAAGACTATGAACAGAT & \#51 \\
\hline & R: AGCACACCCAGTAGCAGTCA & \\
\hline \multirow[t]{2}{*}{ Argl (arginase 1) } & F: GAATCTGCATGGGCAACC & $\# 2$ \\
\hline & R: GAATCCTGGTACATCTGGGAAC & \\
\hline \multirow[t]{2}{*}{ Illo (IL-10) } & F: TGCAGAAAAGAGAGCTCCATC & \#27 \\
\hline & R: TGATCCTCATGCCAGTCAGT & \\
\hline \multirow[t]{2}{*}{ Il6 (IL-6) } & F: GCTACCAAACTGGATATAATCAGGA & \#6 \\
\hline & R: CCAGGTAGCTATGGTACTCCAGAA & \\
\hline \multirow[t]{2}{*}{ Ccl24 (CCL24/Eotaxin 2) } & F: GTGCCTGACCTCCAGAACACT & $\# 2$ \\
\hline & R: GAGGGGATGGTCACAGAATC & \\
\hline \multirow[t]{2}{*}{ Vegfa (VEGFA) } & F: GCAGCTTGAGTTAAACGAACG & \#4 \\
\hline & R: GGTTCCCGAAACCCTGAG & \\
\hline \multirow[t]{2}{*}{$C d 44(\mathrm{CD} 44)$} & F: GTCATCAAACAGAAAGCAAGGAT & \#41 \\
\hline & R: TGTTCAAGTCTTCCACCAAATG & \\
\hline \multirow[t]{2}{*}{ Ki67 (Ki67) } & F: GCTGTCCTCAAGACAАTCATCA & \#80 \\
\hline & R: GGCGTTATCCCAGGAGACT & \\
\hline \multirow[t]{2}{*}{$T g f b 1$ (TGF- $\beta 1)$} & F: TGGAGCAACATGTGGAACTC & \#72 \\
\hline & R: GTCAGCAGCCGGTTACCA & \\
\hline
\end{tabular}

UPL, Universal Probe Library; F, forward; R, reverse.

flow cytometer (BD Biosciences) with FACSDiva v6.1.2 software (BD Biosciences) or FlowJo v10 software (FlowJo, LLC). The following antibodies were used to analyze J774 cells: PE anti-mouse CD301a (MGL1; Miltenyi Biotec $\mathrm{GmbH}$; cat. no. 130-109-216; 1:10 dilution), PE anti-mouse CD206 (BioLegend, Inc.; cat. no. 141706; 1:200 dilution), APC anti-mouse SR-AI/MSR (R\&D Systems, Inc.; cat. no. FAB1797A; 1:1,000 dilution) and PerCP/Cyanine5.5 anti-mouse IA/IE (BioLegend, Inc.; cat. no. 107626; 1:200 dilution). The antibodies were added to the cells and incubated at room temperature for $30 \mathrm{~min}$. PE- and PerCP/Cyanine5.5-conjugated antibodies were excited with a $488 \mathrm{~nm}$ laser, and emitted light was collected via 575/26 and 695/40 filters. The APC-conjugated antibody was excited with a $633 \mathrm{~nm}$ laser, and emitted light was collected via a 660/20 filter.

Arginase activity. Arginase activity was measured in macrophage lysates by using an Arginase Assay kit (cat. no. Z5030047; BioChain Institute, Inc.) according to the manufacturer's protocol with minor modifications. Macrophages were activated as aforementioned and following washing with cold PBS and collection of cells with a cell scraper; a $1 \times 10^{6}$ cells were lysed by adding $100 \mu \mathrm{l}$ of $10 \mathrm{mM}$ Tris- $\mathrm{HCl}$ (pH 7.4) buffer containing $1 \mathrm{mM}$ protease inhibitor cocktail (Merck KGaA) and $0.4 \%$ Triton X-100 (Sigma-Aldrich; Merck KGaA). After centrifugation $\left(14,000 \mathrm{x} \mathrm{g}\right.$ at $4^{\circ} \mathrm{C}$ for $\left.10 \mathrm{~min}\right)$, supernatants were placed into a 96-well plate and used for arginase activity assays. Briefly, $10 \mu \mathrm{l}$ of reaction buffer consisting of the substrate and cofactor was added to $40 \mu \mathrm{l}$ of the sample to allow the conversion of arginine to ornithine and urea by arginase. Arginase activity was proportional to the produced urea concentration, which was measured by adding $200 \mu \mathrm{l}$ of the chromogen. The colored complexes were measured using an Elx808 ${ }^{\mathrm{TM}}$ Absorbance Microplate Reader (BioTek Instruments, Inc.) at a wavelength of $405 \mathrm{~nm}$, and the data were used to calculate the unit arginase activity following the manufacturer's formula: (ODsample-Odblank/ODstandard-ODwater) x10.4, where $1 \mathrm{U}$ is the activity needed to convert $1 \mu \mathrm{Mol}$ of L-arginine to ornithine and urea per minute at $\mathrm{pH} 9.5$ and $37^{\circ} \mathrm{C}$. The experiment was repeated three times.

Nitric oxide synthase (NOS) activity. NOS activity was quantified in cell lysate using an ultrasensitive colorimetric assay NOS kit according to the manufacturer's specifications (cat. no. NB78; Oxford Biomedical Research, Inc.). Due to the activity of NOS, nitric oxide (NO) is generated. Upon addition of the kit components, NO undergoes a series of reactions, and the final product of nitrite is measured using Griess reagent. After $24 \mathrm{~h}$ of incubation with $\mathrm{CM}$, macrophages were washed with PBS and lysed by the addition of RIPA buffer (Thermo Fisher Scientific, Inc.) supplemented with protease inhibitor cocktail (Merck KGaA). After centrifugation 

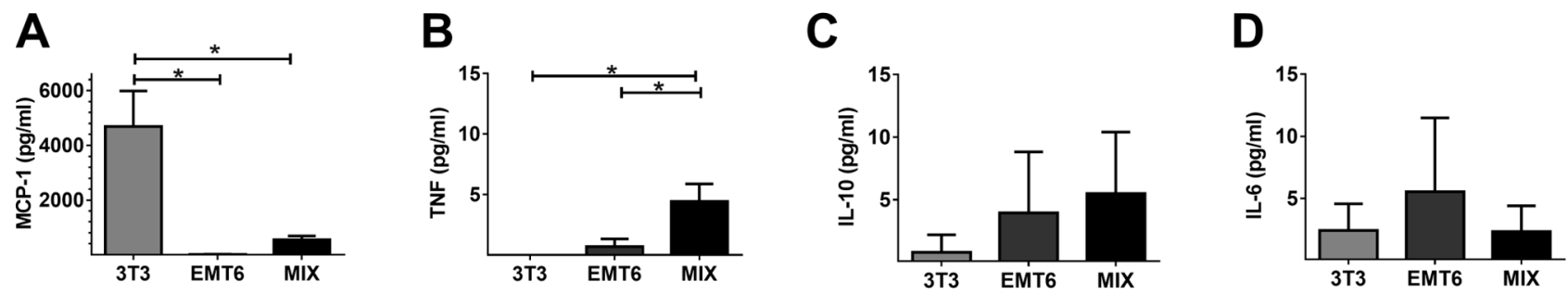

Figure 2. Cytokine concentration in CM. The concentrations of (A) MCP-1, (B) TNF, (C) IL-10 and (D) IL-6 were measured using a BD ${ }^{\mathrm{TM}}$ Cytometric Bead Array. The experiment was repeated three times. Data are presented as the mean \pm SEM. "P $<0.05$ as indicated. CM, conditioned media; 3T3, CM from 3D fibroblast monoculture; EMT6; CM from 3D cancer cell monoculture; MIX, CM from 3D culture of both fibroblasts and cancer cells; MCP-1, monocyte chemoattractant protein 1; TNF, tumor necrosis factor; IL, interleukin.

$\left(14,000 \mathrm{x} \mathrm{g}\right.$ at $4^{\circ} \mathrm{C}$ for $\left.10 \mathrm{~min}\right)$, supernatants were collected, and the protein concentration was measured using a BCA protein assay kit (Thermo Fisher Scientific, Inc.) according to the manufacturer's protocol. Next, the assay was performed as described in the manufacturer's protocol with $30 \mu \mathrm{g}$ of protein. The absorbance was read spectrophotometrically at a wavelength of $540 \mathrm{~nm}$ using an Elx808 Absorbance Microplate Reader (BioTek Instruments, Inc.). The experiment was repeated three times.

Statistical analysis. Statistical analyses were performed in GraphPad Prism v5.01 software (GraphPad Software., Inc.) using one-way ANOVA with the Bonferroni test for multiple comparisons. The experiments were repeated at least three times and the data are presented as the mean \pm SEM. $\mathrm{P}<0.05$ was considered to indicate a statistically significant difference.

\section{Results}

Cytokine secretion in 3D monocultures and the $3 D$ model of breast cancer. Using a CBA assay, the cytokine secretion profiles of $\mathrm{CM}$ collected from the 3D monocultures of cancer cells and fibroblasts and from the 3D model of breast cancer were analyzed. In contrast to the MIX and EMT6 monoculture, the 3T3 monoculture produced a significant quantity of MCP-1 (Fig. 2A). The concentration of TNFa in CM collected from the MIX culture was significantly higher than that in the CM collected from the EMT6 and 3T3 monocultures (Fig. 2B). A larger quantity of IL-10 was isolated from the CM of the MIX and EMT6 cultures compared with the 3T3 monoculture. However, these differences were not significant (Fig. 2C). The EMT6 monoculture exhibited a trend towards a larger quantity of secreted IL-6 compared with other cell cultures (Fig. 2D). The secretion of inflammatory cytokines such as IL-12 and IFN- $\gamma$ was below the detection level in all 3D culture systems (data not shown).

Expression of phenotypic markers in stimulated macrophages. Gene expression in macrophages was analyzed after $12 \mathrm{~h}$ of stimulation with $\mathrm{CM}$ and compared with that of macrophages cultured with CM collected from unseeded silk scaffolds [negative control scaffold (NCS); Figs. 3 and 4]. Nontreated macrophages were used as an additional negative control (NC). Genes characteristic for the M2 phenotype (9) were upregulated in $\mathrm{J} 774$ cells treated with CM from either the MIX culture or the EMT6 monoculture. These genes included those encoding the following proteins: CD11c (Itagx), CD206 scavenger receptor (Mrc1), eotaxin-2 (Ccl24), Fizz-1 (Retnla), arginase-1 (Argl), and IL-10 (Il10) (Fig. 3). The slight increase in Itagx and Argl expression was observed in J774 cells treated with CM collected from the 3T3 monoculture. Medium from the unseeded scaffold did not affect the macrophage phenotype (Fig. 3).

Expression of protumorigenic factors in stimulated macrophages. Stimulated macrophages were analyzed to assess the expression of protumorigenic factors. qPCR showed that the culture of macrophages in CM from the MIX or EMT6 monoculture significantly increased not only $I l 6$ and Tnfa mRNA levels but also Vegfa and Tgfb mRNA levels in comparison with NC (Fig. 4). The level of CD44 was significantly increased in macrophages upon stimulation with CM from EMT6 cells in comparison to stimulation with NC. In addition, increased CD44 expression was found after treatment with the MIX sample. The CM collected from the 3T3 monoculture induced an increase in the Tnfa expression level and slightly increased the $C d 44$ expression level; however, these increases were lower than those induced by MIX and EMT6 CM. CM from none of the tested samples stimulated macrophage proliferation, as indicated by the lack of increased expression of Ki67, a routinely used proliferation marker (36).

Expression of surface markers on stimulated macrophages. Representative results from flow cytometric analysis of macrophages are presented in Fig. 5. Increased expression of CD301a (MGL-1) was observed on macrophages cultured in the presence of CM collected from the MIX model relative to that from macrophages cultured in CM collected from the other 3D cultures. The lowest expression of CD301a (MGL-1) was observed on macrophages treated with CM from the 3T3 monoculture. The expression of the CD206 receptor exhibited the smallest difference in activated macrophages. MHC class II (I-A/I-E) expression was lower on macrophages incubated with CM from the MIX model and EMT6 monoculture than on macrophages stimulated with CM collected from the 3T3 monoculture. The expression of SR-AI/MSR was higher on macrophages treated with CM from the MIX model than on cells incubated with CM from the EMT6 and 3T3 monocultures (Fig. 5).

Activity of arginase in stimulated macrophages. Arginase activity was measured in macrophages using a colorimetric 
A

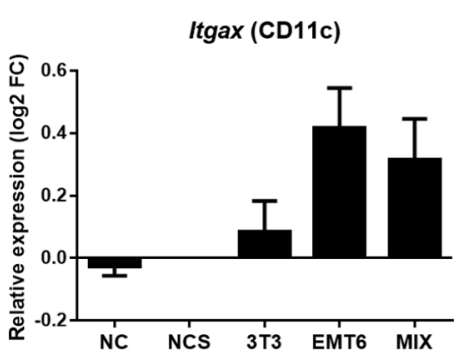

D

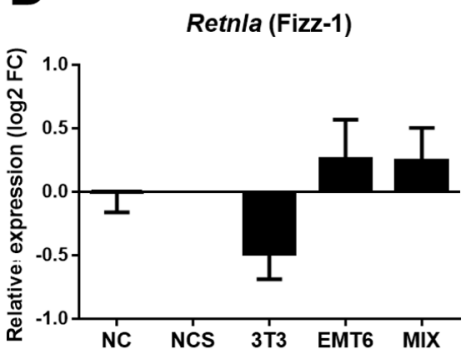

B

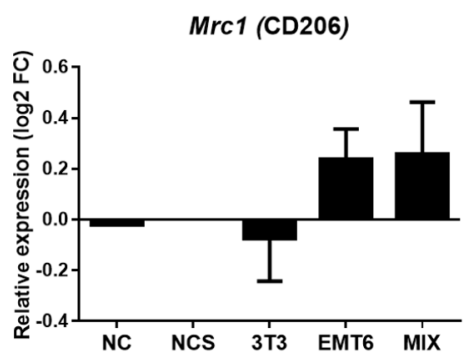

$E$

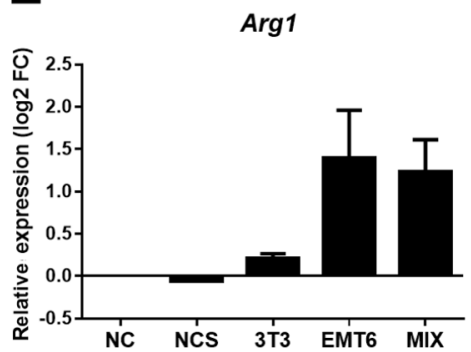

C

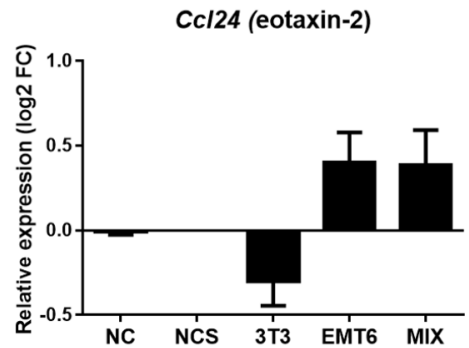

$\mathbf{F}$

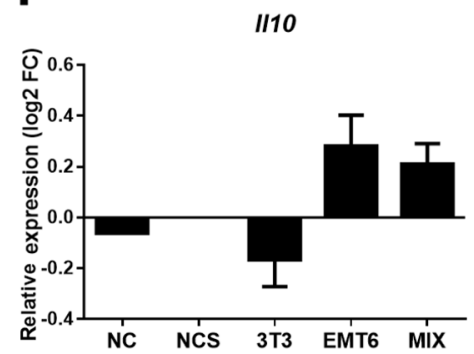

Figure 3. Expression levels of genes characteristic of the M2 phenotype of macrophages upon stimulation with CM. The expression levels of the (A) Itagx (CD11c), (B) Mrcl (CD206), (C) Ccl24 (eotaxin-2), (D) Retnla (Fizz-1), (E) Arg1 (arginase-1), and (F) Ill0 (IL-10) genes were normalized to Tubb ( 3 -tubulin) expression levels. The experiments were repeated at least three times in triplicate. The results are presented as the mean \pm SEM. CM, conditioned medium; 3T3, CM from 3D fibroblast monoculture; EMT6, CM from 3D cancer cell monoculture; MIX, CM from 3D culture of both fibroblasts and cancer cells; NC, fresh medium; NCS, fresh medium from an unseeded scaffold; FC, fold change.

A

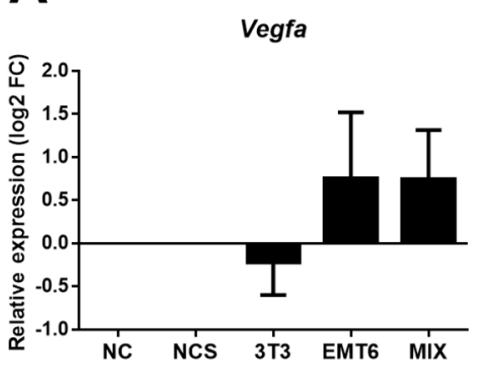

D

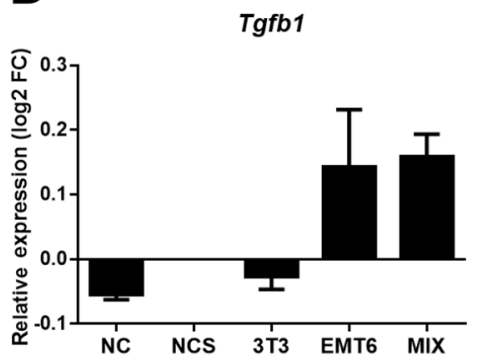

B

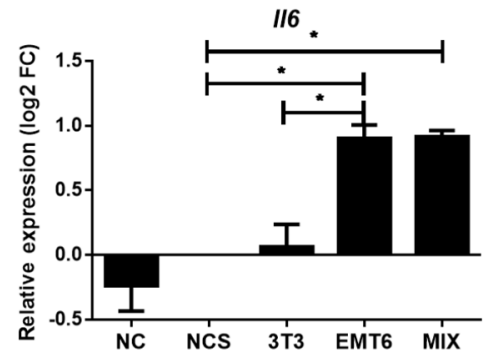

$\mathbf{E}$

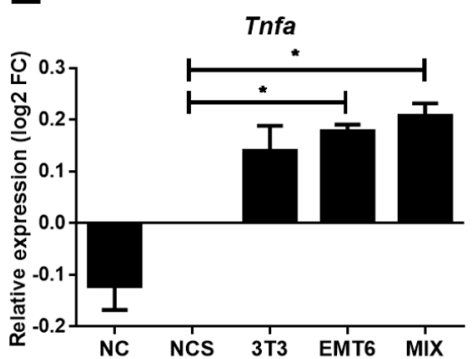

C

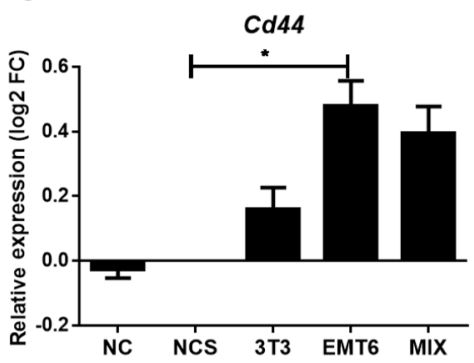

$\mathbf{F}$

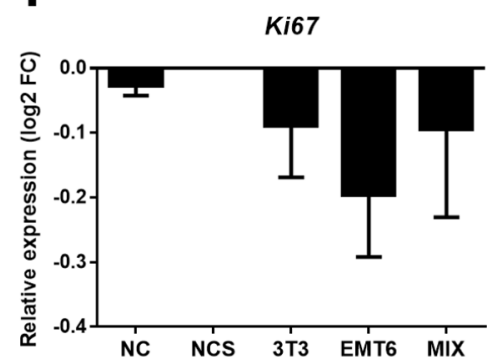

Figure 4. Expression level of genes encoding protumorigenic factors in macrophages upon stimulation with CM from 3D cultures. The expression levels of (A) Vegfa, (B) $I l 6$, (C) Cd44, (D) Tgfbl, (E) Tnfa and (F) Ki67 genes were normalized to Tubb expression levels. Experiments were repeated at least three times in triplicate. The results are presented as the mean \pm SEM. ${ }^{*} \mathrm{P}<0.05$ as indicated. CM, conditioned medium; 3T3, CM from 3D fibroblast monoculture; EMT6, CM from 3D cancer cell monoculture; MIX, CM from 3D culture of both fibroblasts and cancer cells; NC, fresh medium; NCS, fresh medium from an unseeded scaffold; FC, fold change.

arginase assay. The highest arginase activity among CM treated macrophages was demonstrated in cells incubated with CM obtained from the MIX model; the next-highest, in macrophages incubated with CM from the EMT6 monoculture and the lowest, in macrophages incubated with CM from the
$3 \mathrm{~T} 3$ monoculture (Fig. 6). However, the differences were not significant.

Activity of nitric oxide synthase in stimulated macrophages. The intracellular concentration of nitric oxide was measured as 
A

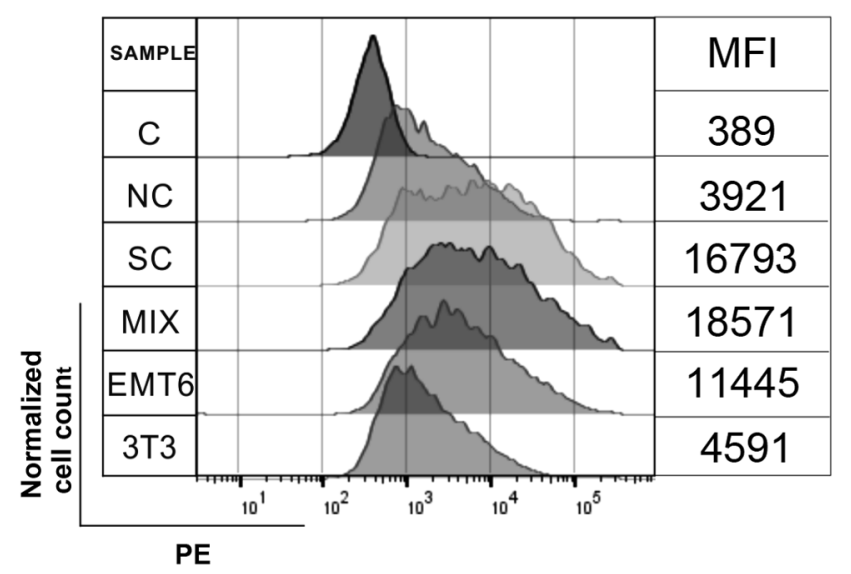

C

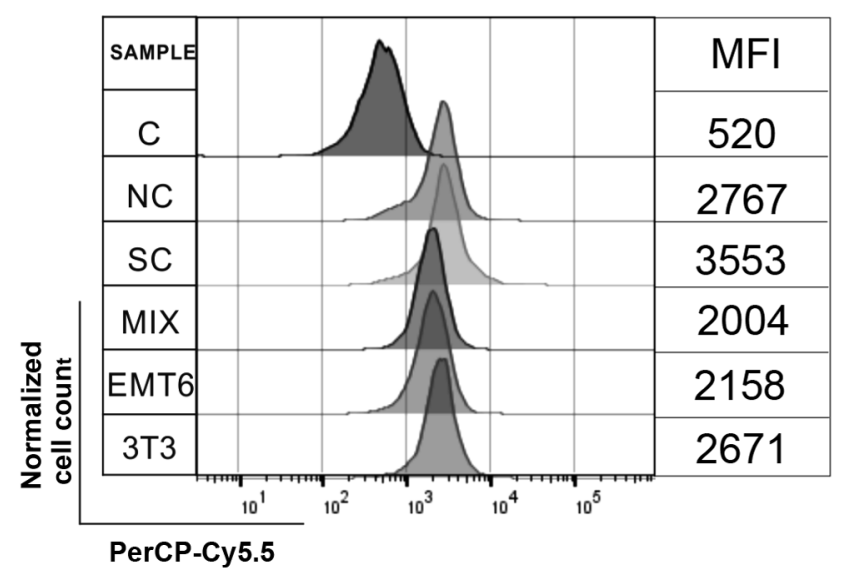

B CD206

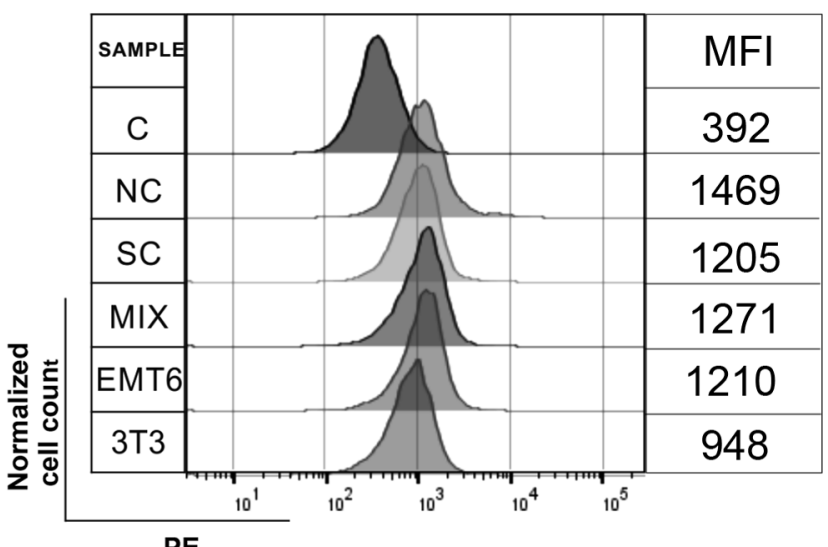

PE

D

CD204 (SR-AI/MSR)

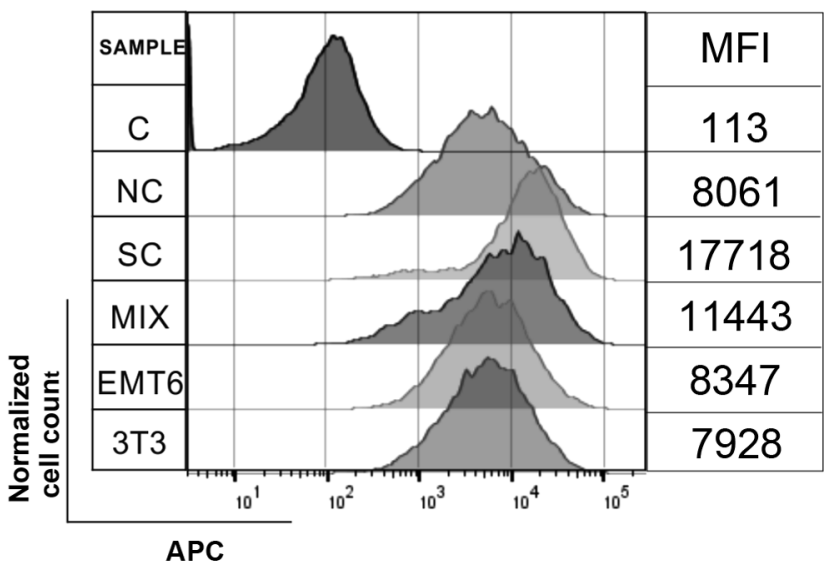

Figure 5. Flow cytometric analysis of macrophages incubated with CMs. The expression of (A) CD301a (MGL1), (B) CD206, (C) I-A/I-E and (D) CD204 (SR-AI/MSR) on representative macrophage histograms. CM, conditioned medium; 3T3, CM from 3D fibroblast monoculture; EMT6, CM from 3D cancer cell monoculture; MIX, CM from 3D culture of both fibroblasts and cancer cells; NC, fresh medium; SC, fresh medium supplemented with IL-4 and IL-6; MFI, mean fluorescence intensity.

a marker of NOS activity. The differences between the macrophages stimulated with $\mathrm{CM}$ were not significant compared with the NC macrophages (Fig. 7).

\section{Discussion}

Currently, in vitro cell culture is the primary tool used to elucidate cell physiology, intercellular-relationships and the impact of various substances on cellular processes. There is a significant difference between the behavior of cells in vivo and those grown in vitro as monolayers (27). As part of the cell surface adheres to the base of the culture dish, cells do not react appropriately to extracellular stimuli. Therefore, certain signaling pathways are artificially silenced. Unfortunately, these pathways are often crucial for the natural response of cells to changes in environmental conditions in terms of biological processes related to metabolism, growth, differentiation and the ability to spread (for example, focal adhesion) (37). Therefore, various approaches have been proposed to generate conditions that more closely imitate nature. As it enables the spatial growth of cells, 3D culture most closely recapitulates the natural microenvironment (38). Silk as a biocompatible material can be used to generate the platforms for developing 3D models of diseases including cancer (33).

A more valuable in vitro model than cell monoculture is coculture that enables a comprehensive understanding of complex intercellular interactions. Coculture is a type of culture in which two or more cell lines are simultaneously combined in the same culture dish. The combination of various cells naturally present in tissue allows for better modeling of a specific tissue environment. In cocultures, cells can adhere directly to each other and contact is mediated by adhesion molecules or receptors. The cells can also communicate by secreting soluble mediators into the environment. In vitro cocultures are used to understand the mechanisms regulating various complex biological processes and to generate the appropriate models for testing modern drugs (39-41).

Our previous studies were focused on building a tumor model that most accurately reflects in vivo conditions $(33,34)$. The natural microenvironment of tumors, in addition to the cancer cells themselves, includes the connective tissue supporting the stroma, immune cells infiltrating the tumor, extracellular matrix and blood vessels, which all participate in cancer nourishment (42). A tumor is a type of 'organ' in which 


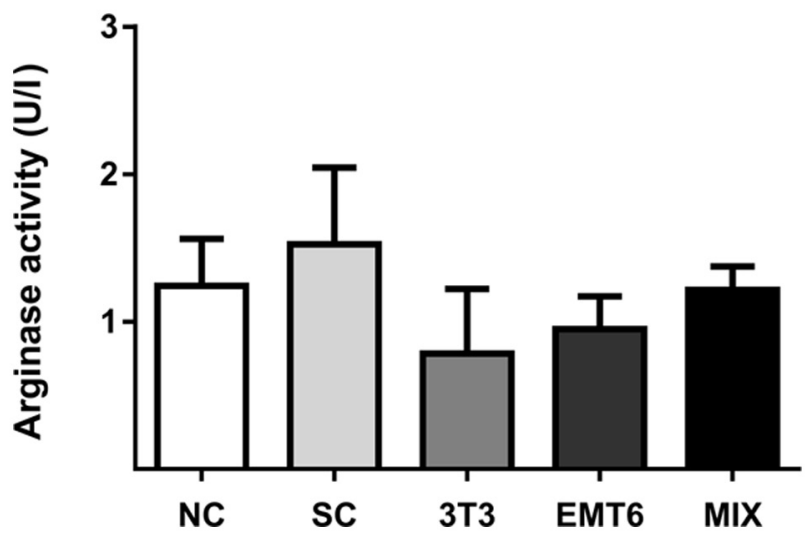

Figure 6. Arginase activity of macrophages activated by incubation in CM. Enzyme activity was determined using an arginase assay kit. The experiment was repeated three times and the results are presented as the mean \pm SEM. CM, conditioned medium; 3T3, CM from 3D fibroblast monoculture; EMT6, CM from 3D cancer cell monoculture; MIX, CM from 3D culture of both fibroblasts and cancer cells; NC, fresh medium; SC, fresh medium supplemented with IL-4 and IL-6.

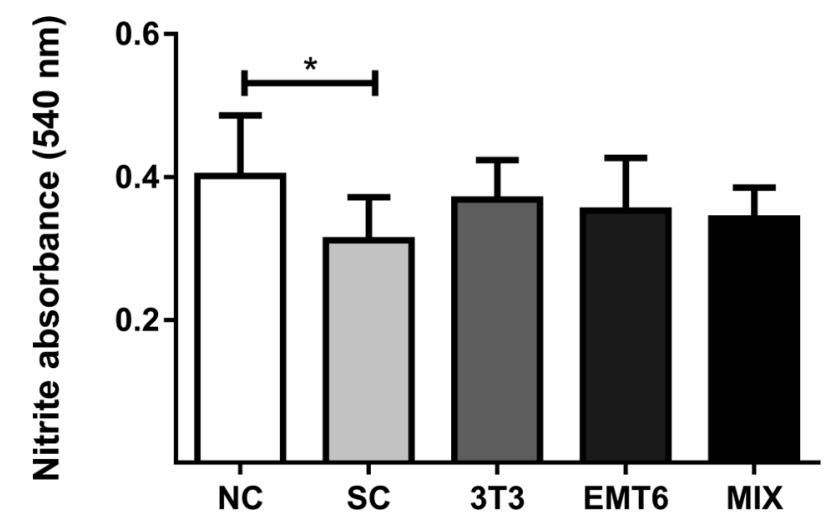

Figure 7. Nitric oxide synthase activity of macrophages activated by incubation with CM. The experiment was repeated three times and the results are presented as the mean $\pm \mathrm{SEM}$. ${ }^{*} \mathrm{P}<0.05$ as indicated. $\mathrm{CM}$, conditioned medium; 3T3, CM from 3D fibroblast monoculture; EMT6, CM from 3D cancer cell monoculture; MIX, CM from 3D culture of both fibroblasts and cancer cells; NC, fresh medium; SC, fresh medium supplemented with IL-4 and IL-6; MFI, mean fluorescence intensity.

cellular, structural and soluble components of the microenvironment participate in controlling biological processes (42). To meet these requirements, an in vitro $3 \mathrm{D}$ model of breast cancer was developed that consists of breast cancer cells and fibroblasts cocultured on a silk scaffold (33). In this cancer model, both tumor dimensionality and cell heterogeneity are considered. The spatial growth of cells enables interactions among cancer cells, cancer-associated cells and the ECM. These interactions induce alterations in the expression levels of ECM components and markers related to EMT and CAF relative to the corresponding levels in cells cultured in $2 \mathrm{D}$ systems and in 3D monoculture systems (33). This model more closely represents the in vivo conditions occurring in the tumor environment than traditional 2D culture and may be beneficial to study tumor biology and to screen drugs (33).

However, as mentioned above, the tumor 'organ' is composed of more than cancer cells and fibroblasts and building a more relevant tumor model is necessary to recapitulate the complexity of the system. To control and better understand the model, it is essential to add individual elements gradually. Among the immune cells recruited to the tumor development site, macrophages are a particularly relevant population (43). Thus, further research is required to examine macrophage behavior in the microenvironment generated by the model. It has been reported that cells that can modify macrophages in the tumor microenvironment are tumor-infiltrating lymphocytes (44), tumor-associated neutrophils (45) and CAFs. As in the present 3D model of breast cancer, fibroblasts acquire the characteristics of CAFs, the application of this model was particularly justified. As previous results demonstrated significant differences between cells cultured under 2D and $3 \mathrm{D}$ conditions and indicated that 3D culture more closely reflects the effects observed in vivo (33), research has continued only on cells that grew on the scaffolds. Indeed, an increasing number of studies, primarily these that investigate the complex interactions in the tumor microenvironment, are focused on only using a 3D model without researching a flat culture system $(30,32)$.

In the current study, levels of cytokines in the CM obtained from cocultured and monocultured cells was measured. This confirmed that the established 3D model of cocultured breast cancer cells and fibroblasts generated a much more complicated microenvironment compared with that of monocultures of each cell line. Measurement of the concentrations of six cytokines: IFN- $\gamma, \mathrm{TNF} \alpha, \mathrm{MCP}-1, \mathrm{IL}-6$, IL-10 and IL-12p70, indicated considerable intersample diversity. For MCP-1 and TNF $\alpha$, the differences were significant. As fibroblasts are the primary source of MCP-1, the lower concentration of this molecule in CM from the MIX system could be a result of a smaller number of fibroblasts in the coculture. As indicated previously, after 1 week of incubation, cancer cells constituted $\sim 70 \%$ and fibroblasts constituted $30 \%$ of the cocultured cells (33). However, the finding that the CM of the MIX sample contained the highest level of TNF $\alpha$ did not result from a simple additive effect of each separate monoculture. This effect likely resulted from the mutual interaction of both cell types. The addition of fibroblasts, which express lower levels of IL-10, to the cancer cells with higher IL-10 expression did not decrease the total level of IL-10 expression; by contrast, the MIX sample showed a trend towards higher expression of IL-10. For IL-6, the addition of fibroblasts to the breast cancer cells resulted in a slight decrease in the total level of IL-6 in the MIX sample compared with the EMT6 cell monoculture. Previous results indicated that fibroblasts after coculture exhibited a significantly higher level of IL-6 mRNA than fibroblasts in 3D monoculture (33), suggesting that fibroblasts could be the primary source of IL- 6 in the MIX sample. However, measuring the mRNA level did not provide information about the amount of protein. It is worth mentioning that the protein levels of the examined factors in the MIX sample indicated the total amount of proteins expressed by both types of cells during coculture. The mRNA levels of the expressed factors examined in the previous study were measured separately in cancer cells and fibroblasts after their separation from coculture (33). In summary, the data presented in both our previous study (33) and the present study indicate that a 3D tumor model generates a different microenvironment than cancer cells and fibroblasts in 3D monoculture. 
The tumor model of the present study was used as a tool to assess tumor biology in terms of macrophage activation. Briefly, CM was collected from 3D monocultures of EMT6, murine fibroblasts (3T3) and a coculture consisting of both cell lines (the 3D breast cancer model, MIX) and used to activate macrophages. Next, the stimulated macrophages were evaluated for possible changes in their activation profiles. To evaluate M1-type activation of macrophages, the level of expression of the major histocompatibility complex type II molecule I-A/I-E was controlled. The activity of NOS was measured alongside the level of TNF $\alpha$ gene expression, which is a proinflammatory cytokine. To monitor M2-type activation, the expression levels of CD206, CD301a and SR-AI/MSR molecules and the enzymatic activity of arginase was measured and the expression levels of the Itgax, Mrcl, Ccl24, RetnIa, Argl and IllO genes were determined. In these macrophages, the expression levels of the Il6, Cd44, Vegfa, and Tgfbl genes were also examined, which encode factors essential for the development of cancer $(15,21)$. Moreover, to monitor the sensitivity of the in vitro cultured macrophages to stimulation, a sensibility control was introduced that consisted of medium supplemented with IL-6 and IL-4. The IL-4 cytokine is frequently used to induce M2 macrophage polarization (46). However, M2 macrophages are heterogeneous groups of cells and can be divided into several subtypes (16). IL-6 has been indicated to serve a significant role in the breast cancer microenvironment (47). Thus, to closely mimic the microenvironment of breast cancer and the characteristics of TAMs, macrophages were also costimulated with IL-6 in the present study. This sensibility control allowed monitoring of the reactivity of macrophages upon stimulation; however, it did not constitute a direct positive control in the experimental model, as it was assumed that the collected CM contained a more complex cocktail of secreted molecules than the sensibility control.

Regardless of whether the CM originated from the EMT6 cell monoculture or the MIX, the expression levels of genes responsible for the $\mathrm{M} 2$ phenotype and genes encoding factors responsible for the progression of cancer were increased similarly in macrophages compared with those treated with control medium. A particularly large increase in the relative expression of the Argl and Vegfa genes was noted. Each of these factors are hallmarks of TAMs/M2 macrophages (9). Moreover, the present results suggested an increase in $\mathrm{Cd} 44$ mRNA levels in macrophages stimulated with CM from the EMT6 and MIX cultures in comparison with those treated with a control. CD44 is a transmembrane receptor for hyaluronic acid that plays an essential role in cell adhesion, cell interactions with the ECM and lymphocyte activation (48). It has been revealed that IL-10 and TNF $\alpha$ increases CD44 expression in monocytes (49). Since CD44 serves a crucial role in maintaining monocytes in the circulation during inflammation and mediating their homing to the inflammation site (50), monocytes/macrophages may be recruited into the tumor microenvironment through that same mechanism. However, this hypothesis requires further study.

As the sensitivity of the methods used to assess RNA and protein levels are different, the macrophage stimulation protocol was modified slightly. CM was collected from three scaffolds of each 3D culture type and the time of macrophage stimulation increased to $24 \mathrm{~h}$. The data indicated that the concentration of secreted soluble factors differed between CM from the 3D cancer model and 3D monocultures, which could influence the activation profile of $\mathbf{J} 774$ macrophages. Indeed, the highest surface expression of the scavenger receptor SR-AI and the galactose-type C-type lectin (CD301a; MGL1) and the lowest surface expression of the I-A/I-E marker was on macrophages cultured with MIX CM. Moreover, it was observed that CM obtained from the 3D tumor model supported the development of the procancer activity of macrophages. J774 macrophages cultured in MIX CM demonstrated reduced NOS enzymatic activity, which was accompanied by increased arginase activity. Although the indicated hallmarks of the M2 phenotype were not limited to macrophages activated by CM derived from cocultured breast cancer cells and fibroblasts, and the observed differences between differently stimulated groups were not always significant, the MIX sample always induced the most pronounced effects on driving macrophages towards M2 polarization.

As the monocultured cells grew faster than the cocultured cells on silk scaffolds (33), media was collected from the $3 \mathrm{D}$ culture systems at different time points. The aim was to compare the influence of CMs collected from a similar total amount of cells from the 3D cancer cell monoculture, fibroblast monoculture and 3D tumor model. Although the tumor model consisted of $\sim 70 \%$ cancer cells and $30 \%$ fibroblasts at the time of CM collection, it was observed that the stimulation of macrophages by MIX CM was, in some cases, similar to (especially at the RNA level) or stronger than the macrophages' response to $\mathrm{CM}$ from the cancer cell monoculture. This finding again indicated that coculture of both cell types generated a novel microenvironment composed of a different cocktail of secreted factors. Under those conditions, it was not possible to determine which of the components was a key factor. It was previously indicated that upon coculture with cancer cells, fibroblasts acquire markers characteristic of CAFs (33). Other studies have demonstrated that fibroblasts (under the influence of cancer cells) secrete factors activating macrophages into TAMs with M2 characteristics $(51,52)$. However, the role of the CAFs in the 3D cancer model regarding macrophage activation requires more advanced study.

The activation profile of macrophages cultured in the presence of conditioned media obtained from the 3D tumor model was changed towards that of a procancer M2 phenotype, in a comparable manner to that suggested in the literature $(21,32)$. Recently, extensive controversy and ambiguity has surrounded the classification and nomenclature of macrophage activation profiles. The precision of the originally proposed distribution into classical and alternative, or M1 and M2, profiles proved to be insufficient (20). Therefore, Murray et al (53) and a large group of experts proposed a new nomenclature for macrophage activation profiles, along with experimental guidelines. This new insight into macrophage activation profiles is based on information about the source of macrophages, the definition of activators, and a consistent group of markers describing the activation of macrophages. Authors are striving in this way to develop standards for various experiments. In this context, the presently described model can be a more precise tool allowing for the assessment of processes regarding TAM activation in the tumor microenvironment than models based only on the use of monocultures in 2D or 3D conditions. 
The present experiments indicated that the 3D coculture of breast cancer cells and fibroblasts, which mimics the conditions in the tumor microenvironment, constitutes a powerful tool for studying relationships between cancer cells and macrophages. The limitation of the applied experimental process is that the macrophages were stimulated with CMs. In such an experimental set-up, only unidirectional interactions can be induced; only factors secreted by cells cultured in a 3D system could influence macrophages. However, mutual interactions are observed in vivo. The next step will be to assess the relationship between the 3D tumor model and macrophages that will be cocultured in the separate counterparts but with paracrine contact between the cancer model and the macrophages. Moreover, a more advanced experimental setup should enable direct contact between cells in the cancer model and macrophages. As the behavior of macrophages is modulated by numerous factors in vivo, the implementation of the present $3 \mathrm{D}$ model of cancer to investigate the interactions between cancer and macrophages in animal studies would be an essential next step. The experience gained in the current work is crucial to the study of a more complex experimental system that allows the examination of interactions between the tumor microenvironment and macrophages. Such a system could also be relevant to the estimation of the effects of stimuli such as chemotherapeutic and immunomodulatory drugs in the tumor microenvironment.

The obtained results are crucial to studying macrophage polarization in a more advanced experimental system that allows the examination of the direct interactions between the tumor microenvironment and macrophages in $3 \mathrm{D}$ coculture. This knowledge is essential for developing antitumor therapy based on a macrophage-oriented approach. Moreover, the present study is an example of the application of silk biomaterial to generate useful tools for in vitro research.

\section{Acknowledgements}

Not applicable.

\section{Funding}

This project was supported by grants from the Greater Poland Cancer Centre [grant nos. 12/2014(71) and 13/2014(72)].

\section{Availability of data and materials}

The datasets used and/or analyzed during the current study are available from the corresponding author on reasonable request.

\section{Authors' contributions}

HDK and MK were responsible for study conceptualization and confirmed the authenticity of the raw data. AG, ED and KS performed the experiments. MK, HDK, AG, ED and $\mathrm{KS}$ wrote the original draft of the manuscript. AG, MK, ED, KS, AM and HDK analyzed and interpreted the data. HDK, MK and AM reviewed and edited the manuscript. All authors read and approved the final manuscript before publication.

\section{Ethics approval and consent to participate}

Not applicable.

\section{Patient consent for publication}

Not applicable.

\section{Competing interests}

The authors declare that they have no competing interests.

\section{References}

1. Ghosh D and Dawson MR: Microenvironment Influences cancer cell mechanics from tumor growth to metastasis. Adv Exp Med Biol 1092: 69-90, 2018.

2. da Cunha BR, Domingos C, Stefanini ACB, Henrique T, Polachini GM, Castelo-Branco P and Tajara EH: Cellular interactions in the tumor microenvironment: The role of secretome. J Cancer 10: 4574-4587, 2019.

3. Barbazan J and Matic Vignjevic D: Cancer associated fibroblasts: Is the force the path to the dark side? Curr Opin Cell Biol 56: 71-79, 2019

4. Prenen $\mathrm{H}$ and Mazzone M: Tumor-associated macrophages: A short compendium. Cell Mol Life Sci 76: 1447-1458, 2019.

5. Anfossi S, Fu X, Nagvekar R and Calin GA: MicroRNAs, regulatory messengers inside and outside cancer cells. Adv Exp Med Biol 1056: 87-108, 2018.

6. Allavena P and Mantovani A: Immunology in the clinic review series; focus on cancer: Tumour-associated macrophages: Undisputed stars of the inflammatory tumour microenvironment. J Clin Exp Immunol 167: 195-205, 2012.

7. Shapouri-Moghaddam A, Mohammadian S, Vazini H, Taghadosi M, Esmaeili SA, Mardani F, Seifi B, Mohammadi A, Afshari JT and Sahebkar A: Macrophage plasticity, polarization, and function in health and disease. J Cell Physiol 233: 6425-6440, 2018.

8. Orecchioni M, Ghosheh Y, Pramod AB and Ley K: Macrophage Polarization: Different gene signatures in M1(LPS+) vs. Classically and M2(LPS-) vs. Alternatively activated macrophages. Front Immunol 10: 1084, 2019.

9. Tamura R, Tanaka T, Yamamoto Y, Akasaki, Y and Sasaki H: Dual role of macrophage in tumor immunity. Immunotherapy 10: 899-909, 2018.

10. Laviron $\mathrm{M}$ and Boissonnas A: Ontogeny of tumor-associated macrophages. Front Immunol 10: 1799, 2019.

11. Poh AR and Ernst M: Targeting macrophages in cancer: From bench to bedside. Front Oncol 8, 49, 2018.

12. Salmaninejad A, Valilou SF, Soltani A, Ahmadi S, Abarghan YJ, Rosengren RJ and Sahebkar A: Tumor-associated macrophages: Role in cancer development and therapeutic implications. Cell Oncol (Dordr) 42: 591-608, 2019.

13. Martinez FO and Gordon S: The M1 and M2 paradigm of macrophage activation: Time for reassessment. F1000Prime Rep 6: $6-13,2014$.

14. Mantovani A, Sica A, Sozzani S, Allavena P, Vecchi A and Locati M: The chemokine system in diverse forms of macrophage activation and polarization. Trends Immunol 25: 677-686, 2004.

15. Yang L and Zhang Y: Tumor-associated macrophages: From basic research to clinical application. J Hematol Oncol 10: 58, 2017.

16. Chen $\mathrm{Y}$ and Zhang X: Pivotal regulators of tissue homeostasis and cancer: Macrophages. Exp Hematol Oncol 6: 23, 2017.

17. Soldano S, Pizzorni C, Paolino S, Trombetta AC, Montagna P, Brizzolara R, Ruaro B, Sulli A and Cutolo M: Alternatively Activated (M2) macrophage phenotype is inducible by endothelin-1 in cultured human macrophages. PLoS One 11: e0166433, 2016.

18. Chavez-Galan L, Olleros ML, Vesin D and Garcia I: Much More than M1 and M2 Macrophages, There are also CD169(+) and TCR(+) Macrophages. Frontiers Immunol 6: 263, 2015.

19. Roszer T: Understanding the mysterious M2 macrophage through activation markers and effector mechanisms. Mediators Inflamm 2015: 816460, 2015 
20. Ruytinx P, Proost P, Van Damme J and Struyf S Chemokine-Induced Macrophage Polarization in Inflammatory Conditions. Front Immunol 9: 1930, 2018.

21. Szebeni GJ, Vizler C, Kitajka K and Puskas LG: Inflammation and Cancer: Extra- and intracellular determinants of tumor-associated macrophages as tumor promoters. Mediators Inflamm 2017: 9294018, 2017.

22. Edin S, Wikberg ML, Rutegard J, Oldenborg PA and Palmqvist R: Phenotypic skewing of macrophages in vitro by secreted factors from colorectal cancer cells. PLoS One 8: e74982, 2013.

23. Kamoshida G, Matsuda A, Sekine W, Mizuno H, Oku T, Itoh S, Irimura T and Tsuji T: Monocyte differentiation induced by co-culture with tumor cells involves RGD-dependent cell adhesion to extracellular matrix. Cancer Lett 315: 145-152, 2012.

24. Neyen C, Pluddemann A, Mukhopadhyay S, Maniati E, Bossard M, Gordon S and Hagemann T: Macrophage scavenger receptor a promotes tumor progression in murine models of ovarian and pancreatic cancer. J Immunol 190, 3798-3805, 2013.

25. Wang X, Zhao X, Wang K, Wu L and Duan T: Interaction of monocytes/macrophages with ovarian cancer cells promotes angiogenesis in vitro. Cancer Sci 104, 516-523, 2013.

26. Hoarau-Vechot J, Rafii A, Touboul C and Pasquier J: Halfway between 2D and animal models: Are 3D cultures the ideal tool to study cancer-microenvironment interactions? Int J Mol Sci 19: 181,2018

27. Xu X, Farach-Carson MC and Jia X: Three-dimensional in vitro tumor models for cancer research and drug evaluation. Biotechnol Adv 32: 1256-1268, 2014.

28. Lv D, Hu Z, Lu L, Lu H and Xu X: Three-dimensional cell culture: A powerful tool in tumor research and drug discovery. Oncol Lett 14: 6999-7010, 2017.

29. Holle AW, Young JL and Spatz JP: In vitro cancer cell-ECM interactions inform in vivo cancer treatment. Adv Drug Deliv Rev 97: 270-279, 2016.

30. Rebelo SP, Pinto C, Martins TR, Harrer N, Estrada MF, Loza-Alvarez P, Cabecadas J, Alves PM, Gualda EJ, Sommergruber W and Brito C: 3D-3-culture: A tool to unveil macrophage plasticity in the tumour microenvironment. Biomaterials 163: 185-197, 2018.

31. Grolman JM, Zhang D, Smith AM, Moore JS and Kilian KA: Rapid 3D extrusion of synthetic tumor microenvironments. Adv Mater 27: 5512-5517, 2015.

32. Tevis KM, Cecchi RJ, Colson YL and Grinstaff MW: Mimicking the tumor microenvironment to regulate macrophage phenotype and assessing chemotherapeutic efficacy in embedded cancer cell/macrophage spheroid models. Acta Biomater 50: 271-279, 2017.

33. DondajewskaE,Juzwa W, Mackiewicz A and Dams-KozlowskaH: Heterotypic breast cancer model based on a silk fibroin scaffold to study the tumor microenvironment. Oncotarget 9: 4935-4950, 2018.

34. Penderecka K, Ibbs M,Kaluzna A,Lewandowska A, Marszalek A, Mackiewicz A and Dams-Kozlowska H: Implementation of a dynamic culture condition to the heterotypic 3D breast cancer model. J Biomed Mater Res B Appl Biomater 108: 1186-1197, 2020.

35. Livak KJ and Schmittgen TD: Analysis of relative gene expression data using real-time quantitative PCR and the 2(-Delta Delta C(T)) method. Methods 25: 402-408, 2001

36. Scholzen T and Gerdes J: The Ki-67 protein: From the known and the unknown. J Cell Physiol 182: 311-322, 2000.

37. Asghar W, El Assal R, Shafiee H, Pitteri S, Paulmurugan R and Demirci U: Engineering cancer microenvironments for in vitro 3-D tumor models. Mater Today (Kidlington) 18: 539-553, 2015.

38. Devarasetty M, Mazzocchi AR and Skardal A: Applications of bioengineered 3D tissue and tumor organoids in drug development and precision medicine: Current and future. BioDrugs 32: 53-68, 2018.
39. Vidmar J, Chingwaru C and Chingwaru W: Mammalian cell models to advance our understanding of wound healing: A review. J Sur Res 210: 269-280, 2017.

40. Xu R and Richards FM: Development of in vitro co-culture model in anti-cancer drug development cascade. Comb Chem High Throughput Screen 20: 451-457, 2017.

41. Gordon JL, Brown MA and Reynolds MM: Cell-Based methods for determination of efficacy for candidate therapeutics in the clinical management of cancer. Diseases 6: 85, 2018.

42. Egeblad M, Nakasone ES and Werb Z: Tumors as organs: Complex tissues that interface with the entire organism. Dev Cell 18: 884-901, 2010.

43. Sanchez LR, Borriello L, Entenberg D, Condeelis JS, Oktay MH and Karagiannis GS: The emerging roles of macrophages in cancer metastasis and response to chemotherapy. J Leukoc Biol 106: 259-274, 2019.

44. de la Cruz-Merino L, Barco-Sanchez A, Henao Carrasco F, Nogales Fernandez E, Vallejo Benitez A, Brugal Molina J, Martinez Peinado A, Grueso Lopez A, Ruiz Borrego M, Codes Manuel de Villena M, et al: New insights into the role of the immune microenvironment in breast carcinoma. Clin Dev Immunol 2013: 785317, 2013.

45. Galdiero MR, Bonavita E, Barajon I, Garlanda C, Mantovani A and Jaillon S: Tumor associated macrophages and neutrophils in cancer. Immunobiology 218: 1402-1410, 2013.

46. Shahbazi MA, Sedighi M, Bauleth-Ramos T, Kant K, Correia A, Poursina N, Sarmento B, Hirvonen J and Santos HA: Targeted reinforcement of macrophage reprogramming toward M2 polarization by IL-4-loaded hyaluronic acid particles. ACS Omega 3: 18444-18455, 2018.

47. Masjedi A, Hashemi V, Hojjat-Farsangi M, Ghalamfarsa G, Azizi G, Yousefi M and Jadidi-Niaragh F: The significant role of interleukin-6 and its signaling pathway in the immunopathogenesis and treatment of breast cancer. Biomed Pharmacother 108: 1415-1424, 2018.

48. Chen C, Zhao S, Karnad A and Freeman JW: The biology and role of CD44 in cancer progression: Therapeutic implications. J Hematol Oncol 11: 64, 2018.

49. Gee K, Lim W, Ma W, Nandan D, Diaz-Mitoma F, Kozlowski M and Kumar A: Differential regulation of CD44 expression by lipopolysaccharide (LPS) and TNF-alpha in human monocytic cells: Distinct involvement of c-Jun N-terminal kinase in LPS-induced CD44 expression. J Immunol 169: 5660-5672, 2002.

50. Xu H, Manivannan A, Crane I, Dawson R and Liversidge J: Critical but divergent roles for CD62L and CD44 in directing blood monocyte trafficking in vivo during inflammation. Blood 112: 1166-1174, 2008.

51. Takahashi H, Sakakura K, Kudo T, Toyoda M, Kaira K, Oyama T and Chikamatsu K: Cancer-associated fibroblasts promote an immunosuppressive microenvironment through the induction and accumulation of protumoral macrophages. Oncotarget, 8 , 8633-8647, 2017.

52. Zhang A, Qian Y, Ye Z, Chen H, Xie H, Zhou L, Shen Y and Zheng S: Cancer-associated fibroblasts promote M2 polarization of macrophages in pancreatic ductal adenocarcinoma. Cancer Med 6: 463-470, 2017.

53. Murray PJ, Allen JE, Biswas SK, Fisher EA, Gilroy DW, Goerdt S, Gordon S, Hamilton JA, Ivashkiv LB, Lawrence T, et al: Macrophage activation and polarization: Nomenclature and experimental guidelines. Immunity 41: 14-20, 2014.

This work is licensed under a Creative Commons Attribution-NonCommercial-NoDerivatives 4.0 International (CC BY-NC-ND 4.0) License. 